Pacific Journal of Mathematics

A CLASS OF SMOOTH BUNDLES OVER A MANIFOLD 


\title{
A CLASS OF SMOOTH BUNDLES OVER A MANIFOLD
}

\author{
JAMES EeLls, JR.
}

1. Introduction. In this paper we illustrate certain constructions of importance in the geometry of smooth manifolds. First of all we prove that a homogeneous space $B$ of a connected Lie group $G$ can always be represented as a homogeneous space of a contractible Lie group $E$, necessarily of infinite dimension in general. In particular, that representation shows that the loop space of $B$ can be replaced effectively by a Lie group of infinite dimension. The construction is a special case of a general theory of differentiable structures in function spaces [4]. Secondly, we examine relations between the Lie algebra of $G$ and that of $E$ (this latter being a Banach-Lie algebra), in case $G$ is compact and semi-simple.

As an application we consider certain differentiable fibre bundles over a smooth (i.e., infinitely differentiable) manifold $X$ having infinite dimensional Lie structure groups. Particular attention is given to the bundles associated with maps of $X$ into a sphere; these bundles are important because they are in natural (Poincare dual) correspondence with certain equivalence classes of normally framed submanifolds of $X$. Using a theory of smooth differential forms in function spaces, we give explicit integral representation formulas for the characteristic classes of these bundles. These formulas provide examples of a residue theory of differential forms with singularities [1] - and express those forms with singularities as forms without singularities in differentiable bundles over $X$.

2. The homogeneous spaces. (A) Let $G$ be a connected Lie group (of finite dimension!), and let $L(G)$ denote its Lie algebra, considered as the tangent space to $G$ at its neutral element $e$. If $K$ is a closed subgroup of $G$, we let $B$ denote the homogeneous space $G / K$ of left cosets of $K$. The coset map $\pi: G \rightarrow B$ is an analytic fibre bundle map [9, § 7].

We now construct an acyclic fibre bundle over $B$; our construction is a variant of Serre's space of paths over $B$ based at a point $[8, \mathrm{Ch}$. IV]. For this purpose we have chosen a special class of paths on $G$ suitable for our applications in $\S 5$. (These path spaces are also of importance in the calculus of variations.)

(B) Let $G$ be given a left invariant Riemann structure, determined by an inner product on $L(G)$. If $\mathscr{T}(G)$ denotes the tangent vector bundle of $G$ with projection map $q: \mathscr{T}(G) \rightarrow G$, then $\mathscr{T}(G)$ has induced Riemann structure. If $u, v$ are tangent vectors at a point

Received June 10, 1959. Research partially supported by the Office of Naval Research. 
$m \in G$, we let $(u, v)_{m}$ denote their inner product, and $|v|_{m}$ denote the length of $v$.

Definition. If $I$ is the unit interval $\{t \in I: 0 \leq t \leq 1\}$, we say that a map $x: I \rightarrow G$ is an admissible path on $G$ if it satisfies the following conditions:

(1) $x(0)=e$, the neutral element of $G$;

(2) $x$ is absolutely continuous in the metric of $G$; then its tangent vector $x^{\prime}(t)$ exists for almost all $t \in I$, and we require that

(3) the tangent map $x^{\prime}: I \rightarrow \mathscr{T}(G)$ is square integrable; i.e., the Lebesgue integral

$$
\int_{0}^{1}\left|x^{\prime}(t)\right|_{x(t)}^{2} d t
$$

is finite. We observe that $x(t)=q \circ x^{\prime}(t)$ for each $t \in I$ for which $x^{\prime}(t)$ exists.

Let $E(G)$ denote the totality of admissible paths on $G$. Using pointwise multiplication and metric defined analogously to (1), it is easily seen that $E(G)$ is a topological (metrizable) group. As in the case of continuous path spaces [8, p. 481], $E(G)$ is a contractible group with contraction $h: E(G) \times I \rightarrow E(G)$ given by $h(x, t) s=x(t s)$.

Let $p: E(G) \rightarrow G$ be defined by $p(x)=x(1)$. Then $p$ is a continuous epimorphism whose kernel is the subgroup $\Omega(G)=\{x \in E(G): x(1)=e\}$ of admissible loops on $G$; thus we have an exact sequence

$$
0 \longrightarrow \Omega(G) \longrightarrow E(G) \stackrel{p}{\longrightarrow} G \longrightarrow 0
$$

of topological groups. If $E(G, K)=\{x \in E(G): x(1) \in K\}$, then $E(G, K)$ is a closed subgroup of $E(G)$, and the composition $\lambda=\pi \circ p: E(G) \rightarrow$ $G \rightarrow B$ is a representation of $B$ as a homogeneous space of $E(G)$, with $E(G, K)$ as fibre over $b_{0}=\pi(K) \in B$.

Proposition. $\lambda: E(G) \rightarrow B$ is a principal $E(G, K)$-bundle.

To prove that it remains (by $[9, \mathrm{p} .30]$ ) to show that there is a local section of $E(G)$ defined in a neighborhood of $b_{0}$; because $\pi$ is a bundle map it suffices to find a neighborhood $V$ of $e$ in $G$ and a section $f$ of $E(G)$ over $V$. We use the Riemann structure of $G$ to obtain a neighborhood $V$ of $e$ such that for any point $m \in V$ there is a unique geodesic segment $x_{m}: I \rightarrow V$ such that $x_{m}(0)=e$ and $x_{m}(1)=m$; then $x_{m}$ is clearly an admissible path, and $f(m)=x_{m}$ is a continuous map of $V$ into $E(G)$ such that $p \circ f(m)=m$ for all $m \in V$.

(C) The following result is an application of a general theory of function space manifolds [4].

THEOREM. Let $G$ be a connected Lie group, and $E(G)$ the space of 
its admissible paths. Then $E(G)$ is an infinite dimensional Lie group modeled on a separable Hilbert space. The map $p: E(G) \rightarrow G$ is an analytic bundle epimorphism.

We recall the principal ideas of that construction. Given $x \in E=$ $E(G)$, the tangent space to $E$ at $x$ is the separable Hilbert space $E(x)$ of maps $u: I \rightarrow \mathscr{T}(G)$ such that

(1) $q \circ u(t)=x(t)$ for all $t \in I$,

(2) $u(0)=0$ (the zero in $L(G)$ ), and

(3) the map $u$ is absolutely continuous with square integrable tangent vector field, and the norm $|u|_{x}$ induced from the inner product (3) below is finite. Thus $E(x)$ is considered as the space of admissible variations of the path $x$. The algebraic operations in $E(x)$ are defined pointwise; i.e., if $u, v \in E(x)$ and $a, b \in \boldsymbol{R}$, then $(a u+b v) t=a u(t)+b v(t)$, where the right member is computed in the tangent space $G(x(t))$. A symmetric, bilinear form in $E(x)$ is defined by

$$
(u, v)_{x}=\int_{0}^{1}\left(u^{\prime}(t), v^{\prime}(t)\right)_{x(t)} d t ;
$$

this is an inner product, for if $(u, u)_{x}=0$, then $\left|u^{\prime}(t)\right|_{x(t)}=0$ for almost all $t \in I$, and the condition that $u$ is admissible then implies $u(t)=0$ for all $t \in I$. We emphasize that each $E(x)$ is complete (by standard $L^{2}$ theory), a property that is used in the theory of differentiation in infinite dimensional linear spaces.

Using the natural correspondence (defined locally) between geodesic segments on $G$ emanating from a point $m$ and tangent vectors in $G(m)$, we can find a neighborhood $U_{x}$ (called a coordinate patch) of $x$ in $E(G)$ which is mapped homeomorphically (by a map $\phi_{x}$ called a coordinate system) onto a neighborhood of 0 in $E(x)$ [4, §3]. In overlapping coordinate patches $U_{x}, U_{y}$ we have a map

$$
\phi_{x y}: \phi_{x}\left(U_{x} \cap U_{y}\right) \longrightarrow \phi_{y}\left(U_{x} \cap U_{y}\right)
$$

defined by $\phi_{x y}(u)=\phi_{y} \circ \phi_{x}^{-1}(u)$, and this map is analytic in its domain of definition. (If $\phi$ is a map of an open subset $U$ of a Hilbert space $E$ into a Hilbert space $F$, then $\phi$ is analytic in $U$ if every $x \in U$ has a neighborhood in which $\phi$ can be expressed by the convergent power series

$$
\phi(x+v)=\phi(x)+\sum_{k=1}^{\infty} P_{\phi}^{k}(x, v) / k !,
$$

where $P_{\phi}^{k}(x, v)$ denotes the $k$ th iterated directional derivative of $\phi$ at $x$ in the direction $v$.) Easy modifications of standard Lie group theory show that the group operation in $E(G)$ is analytic and that $p: E(G) \rightarrow$ $G$ is an analytic homomorphism. 
CoRollary. The fibration $\lambda: E(G) \rightarrow B$ is an analytic bundle map.

(D) REMARK. The inner product (3) is easily seen to provide an analytic Riemann structure on $E(G)$. We note, however, that it is not left invariant on $E(G)$.

Suppose we let $G$ act on $E(G)$ by $T_{g}(x) t=g x(t) g^{-1}$ for all $t \in I$ and $x \in E(G)$. If $G$ is compact and semi-simple and if the inner product (3) is computed using the bi-invariant Riemann metric on $G$ (see our $\S 3 \mathrm{~A})$, then the Riemann structure on $E(G)$ is G-invariant.

3. The Lie algebra of certain path groups. (A) Suppose that $G$ is connected, compact, and semi-simple. Then its Killing form $[7, \S \S 6,11]$ defines a bi-invariant Riemann structure on $G$ (essentially unique); furthermore, the inner product and the bracket in $L(G)$ are related by

$$
([x, y], z)=(x,[y, z])
$$

for all $x, y \in L(G)$. By taking a suitable real multiple of the Killing form we can suppose that the norm induced from the inner product and the bracket in $L(G)$ are related by

$$
|[x, y]| \leq|x||y|
$$

for all $x, y \in L(G)$.

(B) If $e$ also denotes the neutral element of $E(G)$ (so that $e(t)=e$ for all $t \in I)$, then the tangent space $E(e)$ consists of those admissible paths on $L(G)$ starting at 0 ; we introduce the bracket of $u$ and $v$ in $E(e)$ by

$$
[u, v] t=[u(t), v(t)]
$$

for all $t \in I$.

We will call $E(e)$ the Lie algebra of $E(G)$, and henceforth will denote it by $L(E(G))$; note that $L(E(G)=E(L(G))$. Of course the exponential map exp: $L(E(G)) \rightarrow E(G)$ is defined by $(\exp u) t=\exp (u(t))$ for all $t \in I$.

If $|u|_{e}^{2}=(u, u)_{e}$ in the notation of $\S 2(3)$, then the following result, shows that the bracket (3) on $L(E(G))$ is continuous.

Lemma. For any $u, v \in L(E(G)$ we have

$$
|[u, v]|_{e} \leq 2|u|_{e}|v|_{e} \text {. }
$$

Proof. First of all, we note that if $m_{u}=\max \{|u(t)|: t \in I\}$, then $m_{u} \leq|u|_{e}$. Namely, for any $t \in I$ we apply the Schwarz inequality to obtain

$$
|2 u(t)-u(1)|^{2}=\left|\int_{0}^{1} \operatorname{sgn}(t-s) u^{\prime}(s) d s\right|^{2} \leq \int_{0}^{1} \operatorname{sgn}(t-s)^{2} d s \int_{0}^{1}\left|u^{\prime}(s)\right|^{2} d s .
$$


Thus

$$
m_{u} \leq \max \{|2 u(t)-u(1)|: t \in I\} \leq|u|_{e} .
$$

By (2) and the Schwarz inequality in $L(G)$ we find that $|[u, v]|_{e}^{2}$ is bounded by

$$
\begin{aligned}
& \int_{0}^{1}\left\{\left|u^{\prime}(t)\right|^{2}|v(t)|^{2}+2\left|u^{\prime}(t)\right||v(t)||u(t)|\left|v^{\prime}(t)\right|+|u(t)|^{2}\left|v^{\prime}(t)\right|^{2}\right\} d t \\
& \quad \leq m_{v}^{2} \int_{0}^{1}\left|u^{\prime}(t)\right|^{2} d t+2 m_{u} m_{v} \int_{0}^{1}\left|u^{\prime}(t)\right|\left|v^{\prime}(t)\right| d t+m_{u}^{2} \int_{0}^{1}\left|v^{\prime}(t)\right|^{2} d t \\
& \leq 4|u|_{e}^{2}|v|_{e}^{2} .
\end{aligned}
$$

The inequality (4) follows.

REMARK. Unlike the finite dimensional Hilbert-Lie algebra $L(G)$, $L(E(G))$ does not satisfy a relation of the form (1). Thus the bracket in $L(E(G))$ respects its Banach space structure-i.e., $L(E(G)$ is a BanachLie algebra-rather than its structure as a Hilbert space.

(C) Let $p_{*}: L(E(G)) \rightarrow L(G)$ be defined by $p_{*}(u)=u(1)$; clearly $p_{*}$ is a Lie algebra epimorphism, and the inequality

$$
\left|u\left(t_{2}\right)-u\left(t_{1}\right)\right| \leq\left|t_{1}-t_{2}\right|^{1 / 2}|u|_{e} \quad \text { for any } t_{1}, t_{2} \in I
$$

shows that $\left|p_{*}(u)\right| \leq|u|_{e}$ for all $u \in L(E(G))$.

Our next result establishes an infinitesimal analogue of the analytic bundle over $G$ given by Theorem $2 C$.

THEOREM. If $G$ is a connected, compact, semi-simple Lie group, then $p_{*}$ is a continuous Lie epimorphism with kernel $L(\Omega(G))=\Omega(L(G)$ ), the closed ideal of admissible loops on $L(G)$; i.e.,

$$
0 \longrightarrow L(\Omega(G)) \longrightarrow L(E(G)) \stackrel{p_{*}}{\longrightarrow} L(G) \longrightarrow 0
$$

is an exact sequence of Banach-Lie algebras. Furthermore, as Hilbert spaces (but not as Lie algebras), $p_{*}$ induces an orthogonal direct decomposition $L(E(G)) \approx L(\Omega(G)) \oplus M$, where $M$ is a vector space isomorphic to $L(G)$.

Proof. The first statement follows from the algebraic properties of $p_{*}$ and the fact that $p_{*}$ is bounded, and therefore continuous. To prove the second, we define a map $j: L(G) \rightarrow L(E(G))$ by letting $j(x)$ be the linear path $j(x) t=t x$ for each $x \in L(G)$; then $j$ is a linear map of $L(G)$ onto a subspace $M$ of $L\left(E(G)\right.$ ), and $p_{*} \circ j$ is the identity; moreover, $i$ is an isometry, because for any $x, y \in L(G)$,

$$
(j(x), j(y))_{e}=\int_{0}^{1}(x, y) d t=(x, y) .
$$


Note, however, that $M$ is not a subalgebra of $L(E(G))$.

The subspaces $L(\Omega(G))$ and $M$ are orthogonal complements in $L(E(G)$ ), for if $x \in L(G)$ and $v \in L(\Omega(G))$, then

$$
(j(x), v)_{e}=\int_{0}^{1}\left(x, v^{\prime}(t)\right) d t=(x, v(1))-(x, v(0))=0 .
$$

Corollary. The group $\Omega(G)$ of admissible loops on $G$ forms a subgroup of $E(G)$ whose codimension (as a submanifold of $E(G)$ ) equals the dimension of $G$.

REMARK. If $K$ is a closed subgroup of $G$ and if we set $\lambda_{*}=$ $\pi_{*} \circ p_{*}: L(E(G)) \rightarrow L(G) \rightarrow L(G) / L(K)$, then we have an exact sequence of vector spaces

$$
0 \longrightarrow L(E(G, K)) \longrightarrow L(E(G)) \stackrel{\lambda_{*}}{\longrightarrow} L(G) / L(K) \longrightarrow 0 \text {. }
$$

(D) Problem. Consider $L(E(G))$ as a Hilbert space, and form its topological exterior algebra $C^{*}(L(E(G)))$, using the natural inner product on its $p$ th exterior power. The inequality (4) implies that we can construct the Lie algebra cochain complex as in $[7, \S 3]$ and that the differential operator in $C^{*}(L(E(G)))$ is continuous. The elements $\omega \in$ $C^{P}(L(E(G)))$ determine left invariant differential $p$-forms on $E(G)$-an important property because a version of de Rham's Theorem is valid for $E(G)$ (see $\S 5 \mathrm{~A}$ ). What are the relations between the derived cohomology algebras $H^{*}(L(E(G))), H^{*}(L(\Omega(G)))$, and $H^{*}(L(G)) \approx H^{*}(G ; \boldsymbol{R})$ ?

As a first step, because $L(\Omega(G))$ is a closed ideal in $L(E(G))$ we can appeal to our Theorem 3C and Theorem 4 of Cohomology of Lie algebras, G. Hochschild and J-P. Serre, Annals of Math. 57 (1953), 591-603, to obtain the

Proposition. The filtration of $C^{*}(L(E(G)))$ by the ideal $L(\Omega(G))$ determines a spectral sequence such that

$$
E_{2}^{p, q}=H^{p}\left(L(G) ; H^{q}(L(\Omega(G))),\right.
$$

and whose terminal algebra $E_{\infty}$ is the graded algebra associated with $H^{*}(L(E(G)))$, suitably filtered.

4. The bundles over a manifold. (A) Let $B=G / K$ be the homogeneous space of $\S 2 \mathrm{~A}$. Since $E(G)$ is contractible, the fibre bundle $\lambda: E(G) \rightarrow B$ can be interpreted as a universal bundle $[9, \S 19]$ for the infinite dimensional Lie group $E(G, K)$. In particular, by the Classification Theorem for principal bundles we have the

Proposition. If $X$ is a paracompact smooth manifold of finite 
dimension, then the isomorphism classes of smooth principal $E(G, K)$ bundles over $X$ are in natural one-to-one correspondence with the smooth homotopy classes of maps of $X$ into $B$.

In that statement we have made use of the fact that for maps of $X$ into $B$ their classification by homotopy equivalence coincides with classfication by smooth homotopy equivalence.

REMARK. There is a certain uniqueness theorem for universal bundles over $B$, which implies that for any other contractible bundle over $B$ with group $\Gamma$, the homotopy groups of $\Gamma$ are isomorphic to those of $E(G, K)$; see [6, p. 284]. Of course, it follows directly from the homotopy sequence of a bundle and the 5-lemma that the homotopy groups of $E(G, K)$ are isomorphic to those of the loop space of $B$.

(B) Suppose that $B$ is $(n-1)$-connected and that the $n$th homotopy group $\pi_{n}(B)$ is infinite cyclic $(n>1)$; then the group $E(G, K)$ is $(n-2)$ connected, and the connecting homomorphism of the homotopy sequence of the universal bundle of $B$ is an isomorphism of $\pi_{n}(B)$ onto $\pi_{n-1}(E(G, K))$.

Let $\mu: W \rightarrow X$ be an $E(G, K)$-bundle over $X$. Its characteristic class $[9$, p. 178] is the primary obstruction to the construction of a section of the bundle. The condition $n>1$ insures that its structural group is 0-connected, whence the bundle $\mathscr{B}$ of local coefficients (used in defining characteristic classes in general) is simple [9, p. 153]. To orient the bundle is to choose one of the two isomorphism of $\mathscr{B}$ onto the product bundle $X \times Z$. Thus the characteristic class of an oriented $E(G, K)$-bundle over $X$ is a cohomology class $w \in H^{n}(X, Z)$.

It is well known that such a characteristic class can be represented by a transgressive pair of cochains $\left(a^{n}, c^{n-1}\right)$. (A transgressive pair in a bundle consists of a cochain of some sort $c$ on $W$ whose restriction to a fibre is a cocycle of $E(G, K)$, and such that its coboundary $d c=$ $\mu^{*} a$ for some cocycle $a$ of $X$.) Furthermore, the restriction of $c^{n-1}$ to a fibre defines the generator of $H^{n-1}(E(G, K) ; \boldsymbol{Z}) \approx \boldsymbol{Z}$ which is the negative of that determined by the orientation of the bundle.

Let $w_{0}$ be the characteristic class of the universal oriented bundle $\lambda: E(G) \rightarrow B$. Suppose that $\mu: W \rightarrow X$ is induced by the smooth map $f: X \rightarrow B$, and let $g: W \rightarrow E(G)$ be a smooth bundle map covering $f$ $[9, \S 19]$. If $\left(a_{0}, c_{0}\right)$ is a transgressive pair representing $w_{0}$, then $a=f^{*} a_{0}$, $c=g^{*} c_{0}$ is known to be a transgressive pair representing the characteristic class $w$ of $\mu: W \rightarrow X[2, \S 18]$.

5. Representations of the characteristic classes. (A) Let $Y$ be any paracompact smooth manifold modeled on a Hilbert space $E$. A differential $r$-form $\eta$ on $Y$ assigns to each point $y \in Y$ an alternating $r$-linear functional (with real values) on the tangent space $Y(y)$, which is continuous simultaneously in the $r$ variables, using the Hilbert space 
topology in $Y(y)$. In terms of the differentiable structure on $Y$ we can define the exterior algebra $\mathscr{C}^{*}(Y)$ of smooth differential forms on $Y$ and its derived cohomoly algebra $H^{*}\left(\mathscr{C}^{*}(Y)\right.$ ). It is known (an extension of de Rham's Theorem $[4, \S 4])$ that there is a canonical isomorphism of $H^{*}\left(\mathscr{C}^{*}(Y)\right)$ onto $H^{*}(Y ; \boldsymbol{R})$, the singular real cohomology algebra of $Y$.

We remark that this result uses the local Hilbert space structure of $Y$ in two ways:

(1) the square of the norm in $E$ is an analytic function on $E$, which implies that there are sufficiently many smooth functions on $Y$;

(2) there is a natural Hilbert space structure on the $r$ th exterior power of $E$; its completeness is used essentially in the differentiability of differential forms.

We will now give examples of such forms which are transgressive pairs on $E(G, K)$-bundles over $X$.

(B) We have seen in Theorem $2 \mathrm{C}$ that the group $E(G)$ of admissible paths on a connected Lie group $G$ is itself a Lie group modeled on a Hilbert space. Since $E(G)$ is contractible, the general existence theorem quoted in (A) insures that any smooth closed $r$-form $\omega$ on $E(G)$ is the exterior differential of a smooth $(r-1$ )-form $\xi$ (for $r>0$ ). The following result uses a standard homotopy construction to give an explicit formula for $\xi$ in case $\omega$ is the $p^{*}$-image of a form on $G$.

Proposition. Given any smooth closed $r$-form $\omega$ on $G(r>0)$, consider the $(r-1)$-form on $E(G)$ defined as follows: For any $x \in E(G)$ and $r-1$ vectors $u_{1}, \cdots, u_{r-1}$ in the tangent space at $x$, set

$$
\xi(x) \cdot u_{1} \vee \cdots \vee u_{r-1}=\int_{0}^{1}\left\{\omega(x(t)) \cdot x^{\prime}(t) \vee u_{1}(t) \vee \cdots \vee u_{r-1}(t)\right\} d t,
$$

where $x^{\prime}(t)$ denotes the tangent vector to $x$ at $x(t)$, and the bracket in the right member (involving the exterior product $\vee$ ) is computed in the tangent space $G(x(t))$. Then $\xi$ is a smooth $(r-1)$-form on $E(G)$ and $d \xi=p^{*} \omega$.

Proof. The contraction $h: I \times E(G) \rightarrow E(G)$ given by $h(t, x) s=x(t s)$ is simultaneously continuous in the arguments $(t, x)$, and is a smooth function of $x$ for each $t \in I$. Furthermore, for each $x \in E(G)$ the differential $h_{*}(t, x)$ is a square integrable function of $t$; in particular, if $e_{1}$ denotes the unit vector of $I$, then $\left(h_{*}(t, x) \cdot e_{1}\right) s=s x^{\prime}(t s)$ for almost all $x \in I$.

Because the homomorphism $p$ is analytic, the induced form $\omega^{*}=p^{*} \omega$ is a smooth closed $r$-form on $E(G)$ for which

$$
\xi(x)=\left(k \omega^{*}\right) x=\int_{0}^{1} h^{*} \omega^{*}(t, x) \wedge e_{1} d t
$$


exists (as a Lebesgue integral, where the integrand in the right member involves the interior product with $e_{1}$ ). The explicit formula (3) for $\xi(x)$ below shows that $\xi(x)$ is actually an $(r-1)$-covector and that $\xi$ is smooth. Standard reasoning about homotopy operators for differential forms leads to the identity $\omega^{*}=d k \omega^{*}+k d \omega^{*}$, and because $d \omega=0$, we have $d \xi=\omega^{*}$.

Consider the composite map $q=p \circ h: I \times E(G) \rightarrow B$. It is easily checked that $q_{*}(t, x) e_{1}=x^{\prime}(t)$ for almost all $t \in I$, and for any $u$ in the tangent space at $x$ (interpreted as the vector $0 \oplus u$ in the tangent space of $I \times E(G)$ at $(t, x))$ we have $q_{*}(t, x) u=u(t)$. If we take vectors $u_{1}$, $\cdots, u_{r-1}$ as in the hypotheses,

$$
\begin{aligned}
& \xi(x) \cdot u_{1} \vee \cdots \vee u_{r-1}=\int_{0}^{1} h^{*} \circ p^{*} \omega(t, x) \cdot e_{1} \vee u_{1} \vee \cdots \vee u_{r-1} d t \\
& \quad=\int_{0}^{1}\left\{\omega(x(t)) \cdot q_{*}(t, x) e_{1} \vee q_{*}(t, x) u_{1} \vee \cdots \vee q_{*}(t, x) u_{r-1}\right\} d t \\
&=\int_{0}^{1}\left\{\omega(x(t)) \cdot x^{\prime}(t) \vee u_{1}(t) \vee \cdots \vee u_{r-1}(t)\right\} d t .
\end{aligned}
$$

Corollary. Let $\lambda: E(G) \rightarrow B$ be the universal $E(G, K)$-bundle of $\S 2 \mathrm{~B}$. Then for any smooth closed $r$-form $\omega_{0}$ on $B$, the formula (1) with $\omega$ replaced by $\pi^{*} \omega_{0}$ defines a smooth $(r-1)$-form $\xi_{0}$ on $E(G)$ such that $d \xi_{0}=\lambda^{*} \omega_{0}$.

If $i: E(G, K) \rightarrow E(G)$ is the inclusion homomorphism ${ }_{r}$ then we remark that $\eta_{0}=i^{*} \xi_{0}$ is the suspension of $\omega_{0}$ in the sense of $[8, \mathrm{p} .453]$. Applying [8, Cor. 2, p. 469], we obtain the

CoRollary. If $B$ is $(n-1)$-connected and $\pi_{n}(B)$ is infinite cyclic $(n>1)$ and if $\omega_{0}$ is a closed $n$-form representing a generator $v$ of $H^{n}(B ; \boldsymbol{Z})$, then $\left(\omega_{0}, \xi_{0}\right)$ is a transgressive pair representing $v$.

REMark. Suppose that $\mathscr{G}$ is connected, compact, and semi-simple. Then the bi-invariant Riemann structure on $G$ induces an analytic $G$-invariant Riemann structure on $B$. In the preceding corollary a generator $v$ is then represented by a unique harmonic $n$-form $\omega_{0}$; furthermore, $\omega_{0}$ is $G$-invariant, and $\pi^{*} \omega_{0}$ can be expressed as an exterior polynomial in (left invariant) Maurer-Cartan forms on $G$. Thus the generator $v$ is uniquely represented by a transgressive pair $\left(\omega_{0}, \xi_{0}\right)$ where $\omega_{0}$ is harmonic and where $\xi_{0}$ is defined by (1); see $\S 6 \mathrm{~A}$.

(C) We return to the oriented universal bundle $\lambda: E(G) \rightarrow B$, where $B$ is $(n-1)$-connected and $\pi_{n}(B)$ is infinite cyclic $(n>1)$. (These assumptions can be relaxed at the expense of simplicity of exposition.)

Let $X$ be a smooth manifold of finite dimension, and let $\mu: W \rightarrow X$ be a smooth oriented $E(G, K)$-bundle over $X$ with characteristic class $w$. 
Suppose that bundle is induced by a smooth map $f$ of $X$ into $B$, and let $g$ be a smooth bundle map covering $f$ :

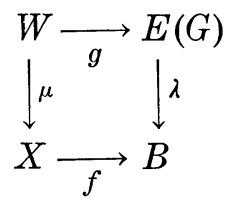

If $\left(\omega_{0}, \xi_{0}\right)$ is a transgressive pair of forms representing the characteristic class $w_{0}$ of $\lambda: E(G) \rightarrow B$ as in (B), then $\omega=f^{*} \omega_{0}, \xi=g^{*} \xi_{0}$ is a transgressive pair representing $w(\S 4 \mathrm{~B})$.

Definition. An admissible partial section of the bundle $\mu: W \rightarrow$ $X$ is a smooth section $\phi$ defined over $X-e(\phi)$, where $e(\phi)$ is a smooth polyhedral subset of $X$ with $\operatorname{dim} e(\phi) \leq \operatorname{dim} X-n$. Admissible partial sections exist because $E(G, K)$ is $(n-2)$-connected. (For example, we can take a smooth locally finite simplicial subdivision $L$ of $X$ and let $L_{*}$ be a dual subdivision; then standard obstruction theory provides a smooth section over a neighborhood of the $(n-1)$-skeleton $L^{(n-1)}$ of $L$ which can be smoothly extended over $X-L_{*}^{(m-n)}$, where $m=\operatorname{dim} X$.)

The following result is an example of the general representation theorem of $[1, \S 4]$; note that the present pair $\left(\omega, \phi^{*} \xi\right)$ satisfies the conditions of Corollary $5 \mathrm{~B}$ of [1]. We will use freely the concepts and results of that paper. As usual in constructing integral formulas for characteristic classes, our method of proof follows that of the GaussBonnet Theorem as given by Chern $[3, \S 2]$ : We first obtain a transgressive pair of forms representing the class; we then appeal to Stokes' Formula to localize and interpret the residue (i.e., the right member of (4) below.

THEOREM. In the above notation, the characteristic class $w$ of the oriented bundle $\mu: W \rightarrow X$ is represented by

$$
w \cdot c=\int_{c} \omega-\int_{\partial c} \phi^{*} \xi
$$

for any admissible partial section $\phi$, where $c$ is any smooth integral $n$-chain on $X$ whose boundary does not intersect $e(\phi)$.

Proof. First of all, $\left(\omega, \phi^{*} \xi\right)$ is an $(\boldsymbol{R}, n)$-pair on $X$ because $\phi$ is admissible, and in $X-e(\phi)$ we have $d\left(\phi^{*} \xi\right)=\phi^{*} d \xi=(\mu \circ \phi)^{*} \omega=\omega$. Secondary, to verify (4) it suffices to do so for the $n$-simplexes of a simplical subdivision $L$ of $X$ (by Corollary 5A of [1]), provided that $e(\phi)$ lies on the $(m-n)$-skeleton of the dual $L_{*}$. Furthermore, in considering its obstruction cocycle we will suppose that $\phi$ is defined only on $L^{(n-1)}$, and then make below a (piecewise smooth) extension to $L^{(n)}-e$, 
where $e$ is a discrete set of points; such an alteration will not change the obstruction class.

Let $b_{\sigma}$ be the barycenter of the oriented $n$-simplex $\sigma$, and let $\sigma_{t}$ be that simplex radially contracted toward $b_{\sigma}$ by the ratio $1:(1-t)$, using an admissible coordinate system on $X$ containing $\sigma$. Let $h$ be a smooth covering homotopy of that contraction. For any $t<1$ and $x$ in $\partial \sigma_{t}$ let $r(x)$ be the radial projection $x$ on $\partial 6$; setting $\phi(x)=h(t, \phi(r(x)))$ defines an extension of $\phi$ over $\sigma-b_{\sigma}$.

Applying Stokes' Formula to the chain $\tau_{t}=\sigma-\sigma_{t}$ we obtain

$$
-\int_{\partial \sigma_{t}} \phi^{* \xi}=\int_{\tau_{t}} \omega-\int_{\partial \sigma} \phi^{* \xi} \text {. }
$$

As $t \rightarrow 1$ the right member approaches the right member of (4) with $c=\sigma$, because $\omega$ is defined on all $\sigma$. To complete the proof of the theorem we will show that as $t \rightarrow 1$ the left member determines the obstruction cocycle.

Since $-\xi$ defines the generator of $\mu^{-1}\left(b_{\sigma}\right)$ by $\S 4 \mathrm{~B}$, we see that (writing $w$ for the obstruction cocycle)

$$
w \cdot \sigma=-\int_{\partial \sigma} \phi^{*} \xi \text {. }
$$

On the other hand, the homotopy $h$ satisfies a Lipschitz condition locally on $\mu^{-1}(\sigma)$ (relative to any metric on $W$ ), whence there is a number $M$ independent of $t$ such that $t<1$ implies

$$
\left|\int_{\phi(\partial \sigma)} \xi-\int_{\phi\left(\partial \sigma_{t}\right)} \xi\right| \leq M|1-t| \text {. }
$$

Using the transformation of integral formula, we find that

$$
\left|w \cdot \sigma+\int_{\partial \sigma_{t}} \phi^{*} \xi\right|=\left|\int_{\partial \sigma} \phi^{*} \xi-\int_{\partial \sigma_{t}} \phi^{* \xi}\right| \leq M|1-t| \text {. }
$$

This shows that as $t \rightarrow 1$ the left member of (5) approaches $w \cdot \sigma$, and formula (4) follows.

6. Spherical maps of a manifold. (A) As an example of the proceding constructions let $G=S O(n+1)$, the rotation group in its usual matrix representation in numerical space $\boldsymbol{R}_{n+1}$. Let $K=S O(n)$, considered as the subgroup of $G$ which acts trivially on the $(n+1)$ th axis of $\boldsymbol{R}_{n+1}$. The unit sphere $S_{n}$ in $\boldsymbol{R}_{n+1}$ is then naturally identified with the homogeneous space $G / K$, and the coset map $\pi: S O(n+1) \rightarrow S_{n}$ represents $S O(n+1)$ as the principal $S O(n)$-bundle of orthonormal $n$-frames on $\left.S_{n}\right] 9$, \& 7]. We will suppose that $S_{n}$ has its usual Riemann structure and is oriented by the coordinate axes in $\boldsymbol{R}_{n+1}$. Henceforth we denote the infinite dimensional Lie group $E(S O(n+1), S O(n))$ by $\Lambda_{n}$. 
Let $\omega_{i j}(1 \leq i<j \leq n+1)$ be a base of Maurer-Cartan forms for the conjugate space of $L(S O(n+1))$; if we let $k(n)$ denote the reciprocal of the volume of $S_{n}$, then the exterior polynomial (the Kronecker Index form $)$ on $S O(n+1)$ given by

$$
\omega_{0}^{*}=k(n) \omega_{1, n+1} \vee \cdots \vee \omega_{n, n+1}
$$

is known to be $S_{n}$-basic (i.e., there is a unique $S O(n+1)$-invariant $n$-form $\omega_{0}$ on $S_{n}$ such that $\pi^{*} \omega_{0}=\omega_{0}^{*}$ ), and thereby represents the harmonic generator of $H^{n}\left(S_{n} ; \boldsymbol{Z}\right)$.

Suppose $n$ is even; then a crucial step in the derivation of the Gauss-Bonnet Theorem [3] for $S_{n}$ establishes that $\omega_{0}$ is part of a transgressive pair in the principal frame bundle of $S_{n}$. If $n$ is odd, then $\omega_{0}$ does not generally have that property. However, for all $n>1$ Proposition 5B gives an explicit transgressive pair in the oriented universal bundle of $S_{n}$, determined entirely by the Kronecker Index form.

(B) If $X$ is a compact, oriented, smooth Riemann manifold of dimension $n+m$, then the isomorphism classes of smooth principal $\Lambda_{n}$ bundles over $X$ play an important role in its geometry, primarily because of the following construction: Let $V$ be a closed, oriented, $m$-dimensional regularly imbedded submanifold of $X$; suppose that $V$ admits a smooth normal $n$-frame in $X$, and let $\phi$ be such a frame field; we will call the pair $(V, \phi)$ a normally framed submanifold of $X$. These have been studied by Kervaire [5, § 1] and Thom [10, Ch. II, 4]. It is known that certain equivalence classes of normally framed $m$-submanifolds of $X$ are in natural one-to-one correspondence with the homotopy classes of maps of $X$ into $S_{n}$ [5, $\left.\S 1\right]$. Combining with the Classification Theorem for $\Lambda_{n}$-bundles, we have the

Proposition. If $X$ is a compact, oriented, smooth Riemann $(n+m)$-manifold, then there is a natural one-to-one correspondence between equivalence classes of normally framed m-submanifolds of $X$ and isomorphism classes of smooth $A_{n}$-bundles over $X$.

Let $(V, \phi)$ be a normally framed $m$-submanifold, and let $i: V \rightarrow X$ be the inclusion map; then since $V$ is closed and oriented (the orientation on $X$ and the frame field $\phi$ determine an orientation of $V$ ) we have a distinguished generator $v_{0} \in H_{m}(V, \boldsymbol{Z})$, which determines a definite homology class $i_{*}\left(v_{0}\right)=v \in H_{m}(X, \boldsymbol{Z})$; Furthermore, $v$ depends only on the equivalence class of $(V, \phi)$. On the other hand, applying a theorem of Thom [10, Théorème II.2], we obtain the

Proposition. In the correspondence of the above proposition, the homology class of a normally framed submanifold is the Poincaré dual of the characteristic class of the oriented $\Lambda_{n}$-bundle associated with it. 
(C) Let $X$ be a smooth manifold of finite dimension. In the study of differential forms with singularities [1] it is important (e.g., in working with exterior products of such forms) to know when a closed $(\boldsymbol{Z}, \boldsymbol{r})$-pair is cohomologous to a pair defined in terms of a transgressive pair (as in Theorem 5C). For example, it is well known that the isomorphism classes of $S O(2)$-bundles over $X$ are (by their characteristic classes) in natural one-to-one correspondence with the elements of $H^{2}(X ; \boldsymbol{Z})$. An easy construction shows that every 2-dimensional integral cohomology class of $X$ can be represented by a transgressive pair in a canonically defined $S O(2)-b u n d l e$ over $X$.

A cohomology class $u \in H^{n}(X ; \boldsymbol{Z})$ is said to be spherical if there is a map $f: X \rightarrow S_{n}$ such that $u=f^{*}(s)$ for some $s \in H^{n}\left(S_{n} ; Z\right)$. The representation theorem $[1, \S 4]$ of cohomology classes by forms with singularities together with our Theorem 5C gives a transgressive integral representation formula for every spherical class of $X$ in a $\Lambda_{n}$-bundle. That bundle is uniquely defined by the homotopy class of $f: X \rightarrow S_{n}$, but is not generally determined by $u$.

Example. Suppose that $X$ has dimension $n$. The Hopf Classification Theorem then implies that the isomorphism classes of smooth $A_{n}$-bundles over $X$ are in natural one-to-one correspondence with the elements of $H^{n}(X ; \boldsymbol{Z})$, the correspondence assigning to each isomorphism class its characteristic class. Theorem 5C gives a transgressive integral representation formula for each element $v$ of $H^{n}(X ; \boldsymbol{Z})$ in a bundle canonically associated with $v$. Of course that fact is significant only for compact manifolds, because $H^{n}(X ; \boldsymbol{Z})=0$ if $X$ is open. On the other hand, it is particularly useful for non-orientable compact manifolds, because then $H^{n}(X ; \boldsymbol{Z})$ has torsion, in which case the singularity of a $(\boldsymbol{Z}, n)$-pair representing $v$ plays an essential role.

If $X$ is orientable and if its Euler characteristic $\chi(X) \neq 0$, then the Gauss-Bonnet Theorem provides a transgressive integral formula for the elements of $H^{n}(X ; \boldsymbol{Z})$ in a finite dimensional bundle over $X$. In general (and for lower dimensional spherical classes) it appears necessary to use infinite dimensional smooth bundles to obtain such a formula.

\section{BIBLIOGRAPHY}

1. C. B. Allendoerfer and J. Eells, On the cohomology of a manifold, Comm. Math. Helv. 32 (1958), 165-179.

2. A. Borel, Selected topics in the homology theory of fibre bundles. Mimeographed Notes. Univ. of Chicago (1954).

3. S. S. Chern, On the curvatura integra in a Riemannian manifold, Annals of Math. 46 (1945), 674-684.

4. J. Eells, On the geometry of function spaces, Sym. Inter. de Top. Alg. Mexico (1958), 303-308. 
5. M. Kervaire, Courbure intégrale généralisée et homotopie, Math. Ann. 131 (1956), 219252.

6. J. Milnor, Construction of universal bundles I, Ann. of Math. 63 (1956), 272-284.

7. Séminaire S. Lie; E.N.S. 1954-5.

8. J-P. Serre, Homologie singulière des espaces fibrés, Ann. of Math. 54 (1951), 425-505.

9. N. E. Steenrod, The Topology of Fibre Bundles, Princeton, 1951.

10. R. Thom, Quelques propriétés globales des variétés différentiables, Comm. Math. Helv 28 (1954), 17-86.

COLUMBIA UNIVERSITY 


\section{PACIFIC JOURNAL OF MATHEMATICS}

\section{EDITORS}

David Gilbarg

Stanford University

Stanford, California

F. H. Brownell

University of Washington

Seattle 5, Washington
A. L. Whiteman

University of Southern Californıa Los Angeles 7, California

L. J. PaIge

University of California

Los Angeles 24, California

\section{ASSOCIATE EDITORS}
E. F. BECKENBACH
T. M. CHERRY
D. DERRY

E. HEWITT
A. HORN
L. NACHBIN
M. OHTSUKA

H. L. ROYDEN

M. M. SCHIFFER
E. SPANIER

E. G. STRAUS

F. WOLF

\section{SUPPORTING INSTITUTIONS}

\author{
UNIVERSITY OF BRITISH COLUMBIA \\ CALIFORNIA INSTITUTE OF TECHNOLOGY \\ UNIVERSITY OF CALIFORNIA \\ MONTANA STATE UNIVERSITY \\ UNIVERSITY OF NEVADA \\ NEW MEXICO STATE UNIVERSITY \\ OREGON STATE COLLEGE \\ UNIVERSITY OF OREGON \\ OSAKA UNIVERSITY \\ UNIVERSITY OF SOUTHERN CALIFORNIA
}

\author{
STANFORD UNIVERSITY \\ UNIVERSITY OF TOKYO \\ UNIVERSITY OF UTAH \\ WASHINGTON STATE COLLEGE \\ UNIVERSITY OF WASHINGTON \\ AMERICAN MATHEMATICAL SOCIETY \\ CALIFORNIA RESEARCH CORPORATION \\ HUGHES AIRCRAFT COMPANY \\ SPACE TECHNOLOGY LABORATORIES \\ NAVAL ORDNANCE TEST STATION
}

Mathematical papers intended for publication in the Pacific Journal of Mathematics should be typewritten (double spaced), and the author should keep a complete copy. Manuscripts may be sent to any one of the four editors. All other communications to the editors should be addressed to the managing editor, L. J. Paige at the University of California, Los Angeles 24, California.

50 reprints per author of each article are furnished free of charge; additional copies may be obtained at cost in multiples of 50 .

The Pacific Journal of Mathematics is published quarterly, in March, June, September, and December. The price per volume (4 numbers) is $\$ 12.00$; single issues, $\$ 3.50$. Back numbers are available. Special price to individual faculty members of supporting institutions and to individual members of the American Mathematical Society: $\$ 4.00$ per volume; single issues, $\$ 1.25$.

Subscriptions, orders for back numbers, and changes of address should be sent to Pacific Journal of Mathematics, 2120 Oxford Street, Berkeley 4, California.

Printed at Kokusai Bunken Insatsusha (International Academic Printing Co., Ltd.), No. 6, 2-chome, Fujimi-cho, Chiyoda-ku, Tokyo, Japan.

PUBLISHED BY PACIFIC JOURNAL OF MATHEMATICS, A NON-PROFIT CORPORATION

The Supporting Institutions listed above contribute to the cost of publication of this Journal, but they are not owners or publishers and have no responsibility for its content or policies. 


\section{Pacific Journal of Mathematics}

\section{Vol. 10, No. $2 \quad$ October, 1960}

Maynard G. Arsove, The Paley-Wiener theorem in metric linear spaces ........

Robert (Yisrael) John Aumann, Acceptable points in games of perfect

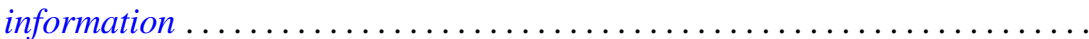

A. V. Balakrishnan, Fractional powers of closed operators and the semigroups generated by them ... . . . . . . . . . . . . . . . . . . . . . . . . . . . . 419

Dallas O. Banks, Bounds for the eigenvalues of some vibrating systems . . . . . 439

Billy Joe Boyer, On the summability of derived Fourier series . . . . . . . . . . . 475

Robert Breusch, An elementary proof of the prime number theorem with

remainder term ...................................

Edward David Callender, Jr., Hölder continuity of $n$-dimensional

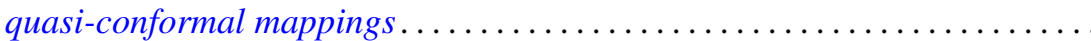

L. Carlitz, Note on Alder's polynomials ......................... 517

P. H. Doyle, III, Unions of cell pairs in $E^{3} \ldots \ldots \ldots \ldots \ldots \ldots \ldots \ldots \ldots \ldots \ldots \ldots . \ldots 21$

James Eells, Jr., A class of smooth bundles over a manifold . . . . . . . . . . . . 525

Shaul Foguel, Computations of the multiplicity function . . . . . . . . . . . . . . 539

James G. Glimm and Richard Vincent Kadison, Unitary operators in

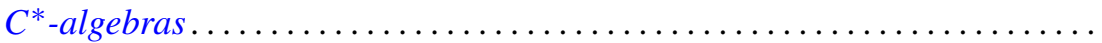

Hugh Gordon, Measure defined by abstract $L_{p}$ spaces . . . . . . . . . . . 557

Robert Clarke James, Separable conjugate spaces ....................

William Elliott Jenner, On non-associative algebras associated with bilinear forms

Harold H. Johnson, Terminating prolongation procedures

John W. Milnor and Edwin Spanier, Two remarks on fiber homotopy type .

Donald Alan Norton, A note on associativity . .

Ronald John Nunke, On the extensions of a torsion module.

Joseph J. Rotman, Mixed modules over valuations rings . . . . .

A. Sade, Théorie des systèmes demosiens de groupoï des . .

Wolfgang M. Schmidt, On normal numbers . .

661

Berthold Schweizer, Abe Sklar and Edward Oakley Thorp, The metrization of

statistical metric spaces

John P. Shanahan, On uniqueness questions for hyperbolic differential

equations

A. H. Stone, Sequences of coverings

Edward Oakley Thorp, Projections onto the subspace of compact operators

L. Bruce Treybig, Concerning certain locally peripherally separable spaces

Milo Wesley Weaver, On the commutativity of a correspondence and a

permutation

David Van Vranken Wend, On the zeros of solutions of some linear complex

differential equations. 